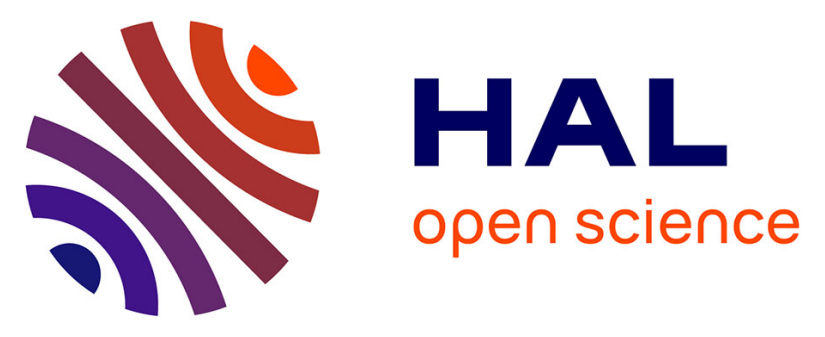

\title{
Inbreeding depression maintained by recessive lethal mutations interacting with stabilizing selection on quantitative characters in a partially self-fertilizing population
}

\author{
Russell Lande, Emmanuelle Porcher
}

\section{To cite this version:}

Russell Lande, Emmanuelle Porcher. Inbreeding depression maintained by recessive lethal mutations interacting with stabilizing selection on quantitative characters in a partially self-fertilizing population. Evolution - International Journal of Organic Evolution, 2017, 71 (5), pp.1191-1204. 10.1111/evo.13225 . mnhn-02265393

\section{HAL Id: mnhn-02265393}

\section{https://hal-mnhn.archives-ouvertes.fr/mnhn-02265393}

Submitted on 9 Aug 2019

HAL is a multi-disciplinary open access archive for the deposit and dissemination of scientific research documents, whether they are published or not. The documents may come from teaching and research institutions in France or abroad, or from public or private research centers.
L'archive ouverte pluridisciplinaire HAL, est destinée au dépôt et à la diffusion de documents scientifiques de niveau recherche, publiés ou non, émanant des établissements d'enseignement et de recherche français ou étrangers, des laboratoires publics ou privés. 
2

${ }_{3}$ Inbreeding depression maintained by recessive lethal mutations 4 interacting with stabilizing selection on quantitative characters ${ }_{5}$ in a partially self-fertilizing population

6 Russell Lande ${ }^{1,2, \dagger}$ and Emmanuelle Porcher ${ }^{3}$

${ }_{8}{ }^{1}$ Department of Life Sciences, Imperial College London, Silwood Park Campus, Ascot, 9 Berkshire SL5 7PY, UK

${ }_{10}$ 2E-mail: r.lande@imperial.ac.uk

${ }_{11}{ }^{3}$ Centre d'Ecologie et des Sciences de la Conservation, Sorbonne Universités UMR 12 MNHN-CNRS-UPMC 7204, 75005 Paris, France

13

14

Running head: Lethal mutations and quantitative genetic variance

KEYWORDS: Alternative equilibria, inbreeding coefficient, inbreeding depression, purging threshold, selective interference, selective facilitation

\section{.}

.

.

${ }^{\dagger}$ Current address: Center for Biodiversity Dynamics, Norwegian University of Science and Technology, N-7491 Trondheim, Norway 
ABSTRACT

The bimodal distribution of fitness effects of new mutations and standing genetic variation, due to early-acting strongly deleterious recessive mutations and late-acting mildly deleterious mutations, is analyzed using the Kondrashov model for lethals (K), with either the infinitesimal model for selfing (IMS) or the Gaussian allele model (GAM) for quantitative genetic variance under stabilizing selection. In the combined models (KIMS and KGAM) high genomic mutation rates to lethals and weak stabilizing selection on many characters create strong interactions between early and late inbreeding depression, by changing the distribution of lineages selfed consecutively for different numbers of generations. Alternative stable equilibria can exist at intermediate selfing rates for a given set of parameters. Evolution of quantitative genetic variance under multivariate stabilizing selection can strongly influence the purging of nearly recessive lethals, and sometimes vice versa. If the selfing rate at the purging threshold for quantitative genetic variance in IMS or GAM alone exceeds that for nearly recessive lethals in $\mathrm{K}$ alone, then in KIMS and KGAM stabilizing selection causes selective interference with purging of lethals, increasing the mean number of lethals compared to K; otherwise, stabilizing selection causes selective facilitation in purging of lethals, decreasing the mean number of lethals.
\end{abstract}


Partially recessive deleterious mutations cause inbreeding depression, or loss of fitness upon matings between relatives, which is a major factor in the evolution of mixed mating systems, particularly mixed self-fertilization and outcrossing which occurs in many plants (Charlesworth and Charlesworth 1987; Charlesworth and Willis 2009) and some hermaphroditic animals (Jarne and Auld 2006). Spontaneous mutations, as well as standing genetic polymorphisms, typically display a strongly bimodal distribution of fitness effects (Dobzhansky 1970; Fudala and Korona 2009; Bell 2010). A class of lethal and sublethal mutations exists which on average in standing variation are nearly recessive; a second class mutations are mildly deleterious and moderately recessive (Simmons and Crow 1977; Willis 1999a,b; Vassilieva et al. 2000; Eyre-Walker and Keightley 2007; Charlesworth and Willis 2009). Homozygous lethal mutations usually act early in development to cause embryonic mortality, while mildly deleterious mutations tend to act later in development, influencing individual growth, survival, and fecundity (Hadorn 1961; Lande et al. 1994; Husband and Schemske 1996; Lande et al. 1994; Bell 2010; Winn et al. 2011). Empirical evidence therefore justifies a life-history model of inbreeding depression due to a combination of recessive lethal mutations affecting embryo survival and mildly deleterious mutations affecting juvenile and adult survival and reproduction.

The Kondrashov (1985) model is often used to describe the genomic evolution of deleterious mutations since with a few reasonable assumptions it accurately describes the population genetic complexities of mixed selfing and outcrossing, especially zygotic disequilibrium (explained below) (Charlesworth et al. 1990; Lande et al. 1994; Kelly 2007; Porcher and Lande 2013, 2016). Based on empirical observations on ferns with a very high inbreeding depression, Ganders (1972) suggested that if nearly all selfed zygotes die before reproduction, then the adult population remains almost completely outcrossed, and selection against recessive deleterious alleles becomes ineffective. Lande et al. (1994) confirmed this idea analytically, showing that with high genomic mutation rates to nearly recessive lethals a process of selective interference among deleterious mutations creates a critical selfing rate, or purging threshold, below which a nearly constant mean number of heterozygous lethals is maintained and above which the equilibrium mean number of lethals decreases dramatically. Kelly (2007) demonstrated 
that selective interference also operates among mildly deleterious moderately recessive mutations if they produce a substantial total inbreeding depression at low or moderate selfing rates.

Mildly deleterious mutations are usually assumed to be unconditionally deleterious (Simmons and Crow 1977; Kondrashov 1985; Charlesworth et al. 1990; Vassilieva et al. 2000; Charlesworth and Willis 2009). However, it is rather difficult to empirically distinguish unconditional mildly deleterious mutations from quantitative genetic variation under stabilizing selection (Charlesworth 2013a,b). Stabilizing selection on a quantitative character with purely additive genetic variance produces allelic effects on fitness that are mildly deleterious and mildly recessive (Wright 1935; Manna et al. 2011), in agreement with general observations on mildly deleterious mutations. In contrast to models with unconditional deleterious mutations, an allele with an additive effect on a quantitative character under stabilizing selection may be either advantageous or deleterious depending on whether the mean phenotype is above or below the optimum, and alleles at different loci with opposite effects on the character may compensate each other in their effects on phenotype and fitness (Fisher 1930, 1958; Wright 1931, 1935, 1969).

Because inbreeding immediately increases the genetic variance of all quantitative characters simultaneously (Wright 1921, 1969), joint stabilizing selection on multiple characters can produce a substantial contribution to the total inbreeding depression, possibly accounting for much or most of the inbreeding depression in fitness due to mildly deleterious mutations (Lande and Schemske 1985). Models of the maintenance of genetic variance in quantitative characters under stabilizing selection in large partially selfing populations demonstrate a similar purging threshold for quantitative genetic variance, which occurs by a different mechanism than that for unconditionally deleterious recessive mutations. For selfing rates below the purging threshold for quantitative genetic variance, the genetic variance remains nearly constant, close to that under random mating, because deviations from Hardy-Weinberg equilibrium are compensated by decreased genic variance and negative linkage disequilibrium, but for selfing rates above the purging threshold, this compensation mechanism breaks down and the total genetic variance becomes greatly reduced. The selfing rate at the purging 
threshold for quantitative genetic variance depends on the total inbreeding depression produced by stabilizing selection on multiple characters (Lande and Porcher 2015).

Here we investigate the evolutionary interaction of the two major components of inbreeding depression by modeling the maintenance of quantitative genetic variance and recessive lethal mutations in a large partially selfing population.

\section{The Models}

In large predominantly outcrossing animal populations, individuals typically carry a few nearly recessive heterozygous lethal or semi-lethal mutations, but the recessive lethal load tends to be somewhat higher in large, long-lived or partially asexual plants (Klekowski 1984, 1988, 1989; Lande et al. 1994; Muirhead and Lande 1997; Scofield and Schultz 2006). With typical karyotypes of many chromosomes, and genomic recombination length of several or many Morgans, even dozens heterozygous recessive lethals in any individual are unlikely to be closely linked, so that to a good approximation alleles at lethal-producing loci can be assumed to segregate independently. Kondrashov's (1985) model of unconditionally deleterious mutations at an infinite number of unlinked loci has been used to model inbreeding depression for fitness due recessive lethal mutations, or due to mildly deleterious mutations (Charlesworth, Morgan and Charlesworth 1990; Lande et al. 1994; Kelly 2007). However, Kondrashov's model assumes that all loci producing detrimental mutations have identical mutation rates and fitness effects, so it can not deal with two components of inbreeding depression unless generalized in some way, and in any case it can not handle compensatory mutations.

Mixed mating systems, such as partial self-fertilization, produce variation among individuals in the degree to which they are inbred. Because inbreeding affects all loci in essentially the same way, variance in the inbreeding coefficient creates zygotic disequilibrium, the non-random association of homozygosity among loci. This presents serious complications for analytical modeling, and the Kondrashov (1985) model for unconditionally deleterious mutations is the only analytical genomic model that accounts exactly for zygotic disequilibrium. Zygotic disequilibrium can be measured by deviations from a Poisson distribution of number of heterozygous lethals in adults (Lande et al. 1994), and by the excess of standardized kurtosis in the distribution of 
quantitative genetic variance, $\kappa$, compared to a normal distribution $(\kappa=3)$. The standardized kurtosis of breeding value in the population is the weighted average fourth central moment within cohorts, divided by the square of the population variance in breeding value (Lande and Porcher 2015).

We model a very large or infinite plant population reproducing by a mixture of self-fertilization and random outcrossing among unrelated individuals. The probability that new zygotes are formed by self-fertilization, $r$, is assumed to be the same for all plants and constant in time. In such a population, every individual can be classified by its selfing age, the number of generations in its immediate past since the last outcrossing event in its genetic lineage or pedigree (Campbell 1986; Schultz and Willis 1995; Kelly 1999a,b; Lande and Porcher 2015).

Kelly (2007) developed an approximate Kondrashov model with selfing age structure, yielding results closely resembling those of the exact Kondrashov model, except near a purging threshold when artifactual limit cycles sometimes appeared. Roze (2015) derived another approximation to the Kondrashov model, allowing zygotic disequilibrium only between pairs of loci; by comparison with the exact Kondrashov model he inferred that for nearly recessive mutations, or with high genomic mutation rates, inbreeding depression is strongly affected by multilocus zygotic disequilbria.

We derive an exact version of the Kondrashov model for nearly recessive lethals (abbreviated as K) structured by selfing age. To reach robust conclusions concerning the total inbreeding depression, we combine the Kondrashov model for lethals (K) with two different models of inheritance for a quantitative character structured by selfing age. The infinitesimal model for selfing (IMS) for a very large but finite population involves an infinite number of unlinked loci with infinitesimal mutational variance. This model extends to inbreeding Fisher's infinitesimal model for an infinite population with no mutation or selection and random or assortative mating (Fisher 1918; Bulmer 1971). IMS accounts for the lower effective population size at higher selfing rates (Wright 1969; Charlesworth and Charlesworth 1995), and the resulting decrease in genic variance maintained by a balance between mutation and random genetic drift in the limit of large population size and small mutation rate. The Gaussian allele model (GAM) assumes an approximately normal distribution of additive allelic effects at each 
of a finite number of linked loci maintained by a balance of mutation, recombination, and stabilizing selection in an infinite population (Kimura 1965; Lande 1975, 1977). In IMS only one stable equilibrium of the genetic variance exists at any selfing rate, but in GAM two stable equilibria exist for selfing rates below the purging threshold, termed the outcrossed equilibrium and the purged equilibrium (Lande and Porcher 2015). These two models of quantitative inheritance combined with the Kondrashov model for lethals are designated respectively as KIMS and KGAM.

The combined models, KIMS and KGAM, neglect the excess kurtosis of breeding values, and the association of lethals with quantitative variation, within selfing age classes, but account for most of the zygotic disequilibrium in the population as a whole. Although we assume that nearly recessive lethal mutations and quantitative genetic variation are selected independently, these two components of inbreeding depression interact because both of them influence the fitnesses and hence the frequency distribution of selfing age classes that forms the framework for their joint evolution.

As for most population genetic models, IMS and GAM are naturally expressed using quantities measured each generation in zygotes before selection. In contrast, the Kondrashov model for lethals, $\mathrm{K}$, is expressed using the numbers of heterozygous recessive lethal mutations in adults. These models can be combined by recalling that homozygous lethal mutations typically act early in development to cause embryonic mortality, while mildly deleterious mutations tend to act late in development, influencing growth, survival and fecundity. Essential features of the IMS and GAM can be preserved when they are combined with K by enumerating genotypes at the subadult stage, after selection on homozygous and heterozygous effects of nearly recessive lethal mutations, but before selection on quantitative characters. The life cycle diagram in Table 1 defines the order of events designated by parenthetic symbols. Unless otherwise stated, the population is measured, and numerical results are graphed, at the subadult stage after selection on lethals but before selection on quantitative characters. 
We model an infinitely large partially self-fertilizing population with no genetic variance in selfing rate, such that each zygote has a probability $r$ of being produced by selffertilization and probability $1-r$ of being produced by outcrossing to an unrelated individual. These probabilities are assumed to be the same for all plants and constant in time. We describe individuals from a lineage self-fertilized for $\tau$ consecutive generations since last outcrossing as being of selfing age $\tau$.

An infinite number of unlinked loci are assumed to undergo irreversible mutation to recessive lethal alleles with total genomic mutation rate $U$. Heterozygous lethal alleles at each locus have viability $1-h$ compared to nonmutant homozygotes, and act independently among loci implying multiplicative fitness effects for individuals with multiple heterozygous lethals.

We define $p_{\tau}(x)$ as the frequency in the population of subadult plants carrying $x$ heterozygous recessive lethal alleles from lineages of selfing age $\tau$, such that $\sum_{\tau=0}^{\infty} \sum_{x=0}^{\infty} p_{\tau}(x)=1$. An individual with $y$ heterozygous lethals reproducing by selffertilization transmits to an offspring $x \leq y$ heterozygous lethal alleles with probability $\left(\begin{array}{l}y \\ x\end{array}\right)\left(\frac{1}{2}\right)^{2 y-x}$, and contributes to outcrossed offspring a gamete carrying $x$ recessive lethal alleles with probability $\left(\begin{array}{l}y \\ x\end{array}\right)\left(\frac{1}{2}\right)^{y}$. Summing over all parental genotypes the recursion equations for transmission of lethals in the selfing lineages are

$$
p_{\tau+1}^{*}(x)=r \sum_{y=x}^{\infty} p_{\tau}^{* * *}(y)\left(\begin{array}{l}
y \\
x
\end{array}\right)\left(\frac{1}{2}\right)^{2 y-x} \quad \text { for } \tau \geq 0
$$

where a triple asterisk ${ }^{* * *}$ denotes parental genotypes after selection on quantitative traits and a single asterisk ${ }^{*}$ denotes offspring genotypes before mutation (eq. 7a).

The gamete pool contributed by all parents is

$$
g(x)=\sum_{\tau=0}^{\infty} \sum_{y=x}^{\infty} p_{\tau}^{* * *}(y)\left(\begin{array}{l}
y \\
x
\end{array}\right)\left(\frac{1}{2}\right)^{y}
$$

and outcrossing by random union of gametes produces offspring of selfing age 0 ,

$$
p_{0}^{*}(x)=(1-r) \sum_{y=0}^{x} g(x-y) g(y)
$$

Mutation, denoted by a double asterisk ${ }^{* *}$, at a diploid genomic rate $U$ per generation 
is described by a Poisson process,

$$
p_{\tau}^{* *}(x)=\sum_{y=0}^{x} p_{\tau}^{*}(x-y) \frac{e^{-U} U^{y}}{y !} \quad \text { for } \tau \geq 0
$$

Selection against heterozygous lethal alleles is described by a selection coefficient $h$ assumed to be identical and to act independently among all loci, so that finally at the subadult stage of enumeration in the next generation, denoted by a prime ', the distribution of numbers of heterozygous lethals by selfing age is

$$
\begin{aligned}
p_{\tau}^{\prime}(x) & =(1-h)^{x} p_{\tau}^{* *}(x) / \overline{\bar{v}} \\
\overline{\bar{v}} & =\sum_{\tau=0}^{\infty} \sum_{x=0}^{\infty}(1-h)^{x} p_{\tau}^{* *}(x)
\end{aligned}
$$

where $\overline{\bar{v}}$ is the mean fitness of juveniles from selection on nearly recessive lethals.

At enumeration in subadults the frequency of selfing age class $\tau$ is

$$
p_{\tau}=\sum_{x=0}^{\infty} p_{\tau}^{\prime}(x)
$$

which is employed in the model of quantitative genetic variation. The mean number of heterozygous lethals in the population is

$$
\bar{x}=\sum_{\tau=0}^{\infty} \sum_{x=0}^{\infty} x p_{\tau}^{\prime}(x) .
$$

\section{QUANTITATIVE GENETIC VARIANCE}

In a randomly mating diploid population the phenotype of an individual for a single character, $z$ is assumed to be composed of a breeding value, $a$ (the sum of additive genetic contributions of alleles at all loci), plus an independent environmental effect, $e$, so that $z=a+e$. Environmental effects are assumed to be normal with mean 0 and variance $E$. Allelic effects are assumed to be purely additive, and hence the genetic variance in breeding value among individuals can be partitioned into two additive components $G=V+C$, where $V$ is the genic variance (twice the variance of allelic effects on each character summed over all loci), and $C$ is twice the total covariance 
of allelic effects among loci within gametes due to linkage disequilibrium (nonrandom association of alleles between loci within gametes). In the absence of selection, inbreeding reduces within-family heterozygosity and additive genetic variance and covariance, and increases additive genetic variance and covariance among families (Wright 1921, 1969, Crow and Kimura 1970). A subpopulation (or cohort) composed of individuals produced by $\tau$ generations of consecutive selfing since the last outcrossing event in their lineage have an inbreeding coefficient $f_{\tau}$ that is uniform (the same for all such individuals). Uniform inbreeding within selfing age cohorts increases purely additive genetic variance by a fraction equal to the inbreeding coefficient (Wright 1921, 1969). The (co)variance of additive genetic effects of alleles from different gametes equals $f_{\tau}$ times the (co)variance of allelic effects, whether or not the alleles are chosen from the same locus. Selfing age cohorts have total genetic variance $G_{\tau}=\left(1+f_{\tau}\right)\left(V_{\tau}+C_{\tau}\right)$ and phenotypic variance $P_{\tau}=G_{\tau}+E$.

To describe stabilizing selection on the individual phenotype for a single quantitative character, $z$, the expected relative fitness of individuals, $W(z)$ is given by a Gaussian function of their deviation from an optimum phenotype, $\theta$,

$$
w(z)=\exp \left\{-\frac{(z-\theta)^{2}}{2 \omega^{2}}\right\} .
$$

Assuming that the mean phenotype of the population is initially at the optimum, $\bar{z}=\theta$, with purely additive genetic variability the phenotype distributions within selfing age cohorts, and in the population as a whole, will always be symmetric with mean phenotype at the optimum, as neither selection nor inbreeding will change the mean phenotype.

Inbreeding depression in fitness due to stabilizing selection on a single quantitative character is likely to be small because the intensity of stabilizing selection typically is moderate or weak (Lande and Arnold 1983, Kingsolver et al. 2001). To produce realistic inbreeding depression in a constant environment we analyze stabilizing selection on multiple quantitative characters. For simplicity, we consider independent stabilizing selection on $m$ identical genetically and phenotypically uncorrelated characters, each with the same genetic and phenotypic variances under the same strength of stabilizing selection. Nearly the same inbreeding depression would be produced by fewer char- 
acters under stronger stabilizing selection. The main results for the maintenance of quantitative genetic variance, including the position of the purging threshold, still apply when the characters have different parameters or are genetically correlated (Lande and Porcher 2015).

Using the normal approximation for phenotypes and breeding values of each character, the mean fitness of a cohort of selfing age $\tau$ is then

$$
\bar{w}_{\tau}=\left(\omega^{2} / \gamma_{\tau}\right)^{m / 2}
$$

where $\gamma_{\tau}=\omega^{2}+P_{\tau}$ measures the strength of stabilizing selection acting on the cohort of selfing age $\tau$ (Lande and Arnold 1983). Stabilizing selection on quantitative characters of subadults changes the lethal heterozygote frequencies in the adult population as a whole by altering the probability distribution of selfing age classes,

$$
p_{\tau}^{* * *}(x)=\frac{\bar{w}_{\tau}}{\overline{\bar{w}}} p_{\tau}(x)
$$

where $\overline{\bar{w}}=\sum_{\tau=0}^{\infty} p_{\tau} \bar{w}_{\tau}$ is the population mean fitness from stabilizing selection on all selfing age cohorts. Summing both sides of this equation over the distribution of numbers of heterozygote lethals within selfing age cohorts gives the cohort frequencies in adults after selection,

$$
p_{\tau}^{* * *}=\sum_{x=0}^{\infty} p_{\tau}^{* * *}(x)=\frac{\bar{w}_{\tau}}{\overline{\bar{w}}} p_{\tau} .
$$

Recursion formulas for completely additive genetic variance under partial selfing are given in Lande and Porcher (2015) for two different models of inheritance. The infinitesimal model for selfing (IMS) assumes an infinite number of loci with infinitesimal mutation. The Gaussian allele model (GAM) assumes a Gaussian distribution of allelic effects at each of a finite number of loci with mutation. Both models assume the loci are unlinked and the population size is very large or practically infinite. The present IMS differs somewhat from that in Lande in Porcher (2015) in that here (1) we did not subdivide the outcrossed age class (selfing age 0) according to parental selfing ages, since numerical computations showed that (in contrast to GAM) the influence of this subdivision is negligible in IMS, and (2) instead of Wright's formula for the mean 
inbreeding coefficient in the population assuming selective neutrality, we use the actual mean inbreeding coefficient in the population to calculate how a larger selfing rate decreases the effective population size and the equilibrium genic variance maintained by mutation (see Appendix). Complete dynamical systems for KIMS and KGAM, with ordering of events and notation as in the life cycle of Table 1, are given by eqs. (1)-(5) for $\mathrm{K}$ in the present paper combined with formulas for either IMS or GAM (Supporting Information; Lande and Porcher 2015), interacting through their joint influence on the selfing age distribution, eqs. (6)-(7).

For numerical analysis it is necessary to truncate the distribution of selfing ages at an upper limit, so that the final class represents individuals selfed consecutively for $L$ generations or longer (Supporting Information). For the Kondrashov model alone, this involves no further approximation because $\mathrm{K}$ is exact even without selfing age structure, and hence can be terminated at any number of selfing ages. However, as for IMS and GAM alone, the combined models may require a large number of selfing age classes for accurate results, as indicated by small excess kurtosis in the population at selfing rates below the purging threshold for quantitative genetic variance. For most parameter values, using 50 to 100 selfing age classes produced nearly the same results as with more age classes.

\section{TOTAL INBREEDING DEPRESSION AND ITS COMPONENTS}

Prout $(1965,1969)$ showed for a complex life cycle with selection at multiple stages that fitness must be measured starting at the beginning of the life cycle with unselected zygotes. The frequencies of selfing age cohorts in zygotes before selection are

$$
\begin{aligned}
p_{0}^{o} & =1-r \\
p_{\tau+1}^{o} & =r p_{\tau}^{* * *} \quad \text { for } \quad \tau=0,1,2, \ldots
\end{aligned}
$$

The viability of selfing age class $\tau$ after selection on lethals but before selection on quantitative traits can be written, and the population viability after selection on lethals 
(eq. 4b) can be rewritten, as

$$
\begin{aligned}
\bar{v}_{\tau} & =\frac{1}{p_{\tau}^{o}} \sum_{x=0}^{\infty}(1-h)^{x} p_{\tau}^{* *}(x) \\
\overline{\bar{v}} & =\sum_{\tau=0}^{\infty} p_{\tau}^{o} \bar{v}_{\tau} .
\end{aligned}
$$

The mean fitness of selfing age class $\tau$ through the complete life cycle, neglecting any association between lethal mutations and quantitative variation within selfing age classes, is then simply $\bar{v}_{\tau} \bar{w}_{\tau}$. From these formulas it can be shown that the mean total fitness in the population is

$$
\overline{\bar{v}} \overline{\bar{w}}=\sum_{\tau=0}^{\infty} p_{\tau}^{o} \bar{v}_{\tau} \bar{w}_{\tau}=\sum_{\tau=0}^{\infty} \sum_{x=0}^{\infty}(1-h)^{x} p_{\tau}^{* *}(x) \bar{w}_{\tau} .
$$

The total inbreeding depression in the population, $\delta$, is one minus the ratio of mean fitness of selfed individuals divided by the mean fitness of outcrossed individuals,

$$
\delta=1-\frac{\sum_{\tau=1}^{\infty} p_{\tau}^{o} \bar{v}_{\tau} \bar{w}_{\tau}}{\left(1-p_{0}^{o}\right) \bar{v}_{0} \bar{w}_{0}}
$$

Components of the total inbreeding depression, due to nearly recessive lethal mutations and stabilizing selection on on quantitative genetic variation, can be defined as

$$
\delta_{v}=1-\frac{\sum_{\tau=1}^{\infty} p_{\tau}^{o} \bar{v}_{\tau}}{\left(1-p_{0}^{o}\right) \bar{v}_{0}}
$$

$$
\delta_{w}=1-\frac{\sum_{\tau=1}^{\infty} p_{\tau} \bar{w}_{\tau}}{\left(1-p_{0}\right) \bar{w}_{0}}
$$

and it can be shown that these combine to compose the total inbreeding depression as usual for a structured life cycle (Lande et al. 1994), $1-\delta=\left(1-\delta_{v}\right)\left(1-\delta_{w}\right)$. These formulas for inbreeding depression can be evaluated for selfing rates between zero and one, $0<r<1$.

\section{PREVALENCE OF MULTIVARIATE STABILIZING SELECTION}

Stabilizing selection on quantitative characters was classically believed to be prevalent in natural populations (Fisher 1930, 1958; Wright 1931, 1935, 1969; Haldane 1954; 
Simpson 1953). Significant stabilizing selection has frequently been observed in natural populations (Lande and Arnold 1983; Kingsolver et al. 2001), but often it is weak and/or fluctuating in time (Lande and Shannon 1996; Siepielski et al. 2009; Engen et al. 2012; Chevin et al. 2015). It is therefore important to realize that directional selection (as defined by Lande and Arnold 1983) also reduces quantitative genetic variance, and hence that, with a stationary distribution of environmental states, randomly fluctuating directional selection produces a net effect of stabilizing selection on quantitative characters (Lande 2007, 2008; McGlothlin 2010).

In multivariate analyses the first few principle components often contain the great majority of the total variance, especially for sets of positively correlated characters such as morphological traits, but this applies to a lesser extent for sets of characters that are less closely integrated (Wright 1968; Lande and Arnold 1983; Blows 2006; Martin and Lenormand 2006; Arnold et al. 2008; Kirkpatrick 2009). Regarding a complex organism as a whole, many nearly independent combinations of morphological, behavioral, and physiological characters must be under stabilizing selection. Furthermore, the strength of stabilizing selection on characters tends to be inversely related to their phenotypic and genetic variances, so the effective number of characters under appreciable stabilizing selection is likely to be considerably larger than the number of significant phenotypic principal components.

\section{HIGH GENOMIC MUTATION RATES TO LETHALS}

Large-scale experiments on Drosophila produced estimates of $U=0.02$ and $h=0.02$ (Simmons and Crow 1977). Because plants lack a germ line, mutations can accumulate during many mitotic cell divisions between meioses, leading to high genomic mutation rates per generation, which may be associated with large adult size, long generations and vegetative reproduction (Klekowski 1984, 1988, 1989; Lande et al. 1994; Muirhead and Lande 1997; Scofield and Schultz 2006). Observations on spontaneous recessive chlorophyll deficiencies occurring at about 300 loci, and scaling these up to 10,000 genomic loci mutating to recessive lethals (Simmons and Crow 1977; Bell 2010) produced estimates of about $U=0.02$ for three annuals, barley, buckwheat and monkey flower, and an order of magnitude higher, $U=0.2$, in mangrove trees (Klekowski and Godfrey 
1989; Willis 1992; Lande et al. 1994).

Perennial plants often set relatively few seeds on experimental self-pollination in comparison to outcrossing, implying either a high early inbreeding depression (EID) causing embryo mortality or late-acting self-incompatibility (LSI) (Seavey and Bawa 1986; Gibbs 2014). LSI is often incomplete and/or variable in expression (Gibbs 2014), and in some species both EID and LSI may occur together (Dorken and Husband 1999; Hao et al. 2012). Extremely high EID occurs in conifer trees which generally are selfcompatible and easily studied because embryo mortality produces an empty seed. For example coastal Douglas fir and other conifer trees have been estimated to harbor a mean of about 10 heterozygous lethal mutations per individual (Sorensen, 19691973 ; Franklin 1971; Bishir and Namkoong 1987). Perennial herbs of the genus Stylidium are fully self-compatible, with the average individual heterozygous for about 20 earlyacting recessive lethals and some populations harboring mean numbers of heterozygous lethals up to 100 (Burbridge and James 1991). Assuming near recessivity of lethals, with $h=0.02$ as estimated from Drosophila (Simmons and Crow 1977), these large mean numbers require high genomic mutation rates to lethals. At mutation-selection balance under random mating (or for selfing rates below the purging threshold for lethals) the mean number of heterozygous lethals in mature plants is $\bar{x}=U(1-h) / h$, so that with $h=0.02$ values of $\bar{x}=10,20$ or 100 corresponds to $U \approx 0.2,0.4$ or 2 . Similarly high values of $U$ have been estimated for a variety of woody perennial species, based on apparent EID (Scofield and Schultz 2006), but in most species the potential role of LSI has not been investigated. Direct evidence of high genomic mutation rates to recessive lethals, up to $U=1$ or larger, comes from studies of spontaneous partially recessive lethals produced during vegetative growth, estimated by comparing seed set from autogamous vs. geitonogamous selfing (Bobiwash et al. 2013).

\section{Results and Discussion}

To analyze the interaction of the two major components of total inbreeding depression corresponding to the bimodal distribution of fitness effects, we modelled nearly recessive lethal mutations and stabilizing selection on quantitative characters acting, respectively, early and late in individual development. Strong interactions occur with 
high genomic mutation rates to lethals and stabilizing selection on many characters, producing a high total inbreeding depression at equilibrium under low selfing rates, as often observed in perennial plants.

\section{ALTERNATIVE EQUILIBRIA}

We discovered that at a high genomic mutation rate to nearly recessive lethals $(U=0.5$, $h=0.02)$, the Kondrashov model for lethals $(\mathrm{K})$ by itself produces two alternative stable equilibria with different mean numbers of heterozygous lethals for selfing rates in the range $0.320 \leq r \leq 0.417$, as in Fig. 1A. The higher equilibrium mean number of lethals is found by starting at equilibrium under complete outcrossing $(r=0$, a Poisson distribution of heterozygous lethals with mean $\bar{x}=U(1-h) / h)$, and increasing the selfing rate in successive increments using as the initial condition for each new value of $r$ the equilibrium distribution of lethals from the previous value of $r$; the higher equilibrium is also reached from the initial condition of zero lethals at all selfing rates. The lower equilibrium mean number of lethals is found by starting at equilibrium under complete selfing $(r=1$, a Poisson distribution of heterozygous lethals with mean $\bar{x}=2 U(1-h) /(1+h))$, and decreasing the selfing rate in successive increments with the initial condition the equilibrium from the previous value of $r$. Lande et al. (1994) developed the Kondrashov model for lethals and investigated the genomic mutation rates to lethals $U=0.02,0.2,1$ with $h=0.02$, using both no lethals and equilibrium under complete outcrossing as initial conditions for all selfing rates, this missed alternative equilibria that exist for $U=0.5$ (Fig. 1A), and for also $U=1$ in a very narrow range of selfing rates $0.516 \leq r \leq 0.520$.

[Fig. 1 about here]

Using the Kondrashov model for lethals structured by selfing age, Fig. 1B,C illustrate the distributions of number of heterozygous lethals per individual, and the corresponding frequency distributions of selfing age, at the two alternative equilibria for $U=0.5$ for selfing rate $r=0.4$. At the higher equilibrium, selective interference among a large mean number of lethals prevents purging and maintains a high 
inbreeding depression which produces a young distribution of selfing ages. The lower equilibrium has purged most of the lethals, maintaining a greatly reduced inbreeding depression and an older distribution of selfing ages.

Because we assume weak stabilizing selection on each character, model $\mathrm{K}$ alone (no quantitative characters, $m=0$ ) gives results for all variables (except genetic variance) close to those for a single character $(m=1)$. This can be seen for the mean number of heterozygous lethals, $\bar{x}$, by comparing Fig. 1A $(m=0)$ with Figs. 2 and 3 middle panel top row ( $m=1$, black lines). We therefore do not plot $m=0$ in subsequent figures, instead focusing on results with increasing number of characters producing higher inbreeding depression from stabilizing selection.

Alternative stable equilibria for nearly recessive lethals still exist when evolving jointly with quantitative variation in a small or moderate number of characters under weak stabilizing selection. In KIMS (Fig. 2) with $U=0.5$, inclusion of up to 10 characters produces two alternative mean numbers of lethals resembling that in $\mathrm{K}$ alone for intermediate selfing rates. With 25 characters these alternative equilibria merge into a single equilibrium, but with 50 or 100 characters new alternative equilibria arise for selfing rates around the purging threshold for lethals.

For all parameter values we examined across the entire range of selfing rates, in KIMS at most two alternative stable equilibria occurred at a given selfing rate. For $U=0.5$ and $m=1$ or 10 , the alternative equilibria at intermediate selfing rates are clearly created by K. However, alternative equilibria appearing at selfing rates around the purging threshold for lethals, particularly at high $U$ and large $m$, are caused by the interaction of numerous lethals with stabilizing selection on many quantitative characters. Fig. 2 shows that alternative stable equilibria for the mean number of lethals exist over some ranges of selfing rates for all values of $U$ illustrated, but not at the lower value of $U=0.02$ (not shown). In all cases where alternative stable equilibria exist at a given selfing rate, the equilibria differ far more in mean number of lethals than in the mean quantitative genetic variance.

[Fig. 2 and Fig. 3 about here: align horizontally on facing pages] 
In GAM alone, without lethals, a purged equilibrium exists at all selfing rates $(r>0)$ caused by the progressive buildup of genic variance and negative covariance due to linkage disequilibrium between loci produced by stabilizing selection in longselfed lineages (Lande and Porcher 2015). This creates a large segregation variance in selfed or outcrossed $F_{2}$ progeny following $F_{1}$ outcrossing, leading to a negative inbreeding depression (also called outbreeding depression) in the population. The purged equilibrium with low total genetic variance in quantitative traits is stable even at intermediate or low selfing rates because strong outbreeding depression shifts the selfing age distribution toward older selfing lineages, maintaining a stable core of the population composed of long-selfed lineages. With the inclusion of recessive lethals in KGAM, this also produces a low mean number of lethal mutations in the long-selfed lineages, so that both quantitative variation and mean lethals remain purged across a wide range of selfing rates, as seen in Fig. 3.

Thus at low or intermediate selfing rates, up to two alternative stable equilibria can occur in KIMS and up to three alternative stable equilibria can exist in KGAM. At high selfing rates in both KIMS and KGAM only the purged equilibrium exists, with low mean number of lethals and low quantitative genetic variance.

\section{INTERACTION OF LETHALS AND QUANTITATIVE GENETIC VARIANCE}

In KIMS (Fig. 2) the crossing of lines of different colors (for different numbers of characters) indicate that the interaction of the two components of inbreeding depression, from nearly recessive lethals and quantitative genetic variance, are more complex than suggested by the simple notion of selective interference. These two components of inbreeding depression display both interference and facilitation in their mutual purging processes. For example, at the lowest genomic mutation rate to lethals shown, $U=0.2$, at selfing rates below about 0.4 increasing numbers of characters up to $m=50$ increases the equilibrium mean number of lethals, demonstrating that stabilizing selection on quantitative characters interferes with purging of lethals; but for $m=100$ at intermediate selfing rates $0.2<r<0.4$ the equilibrium mean number of lethals is actually less that for the Kondrashov model alone, demonstrating that selection on the quantitative characters facilitates purging of lethals. Facilitation of purging reces- 
sive lethal mutations by stabilizing selection on quantitative traits becomes the main pattern of interaction with higher $m$ and $U$ (Fig. 2 top row). Stabilizing selection on quantitative characters facilitates purging of recessive lethals when the purging threshold for quantitative variation alone is below the purging threshold for lethals alone. This happens because of the shift in the age distribution toward older selfing ages that occurs during the purging of quantitative variation, which in turn initiates the purging of recessive lethals. In contrast, recessive lethals have relatively little impact on the purging of quantitative genetic variance by selfing in KIMS, only slightly smoothing or perturbing the otherwise abrupt purging threshold for quantitative variation (Fig. 2 second row).

Fig. 4 illustrates for KIMS at a given selfing rate that increasing the number of characters under stabilizing selection (increasing the inbreeding depression due to mildly deleterious mutations) initially shifts the equilibrium distribution of selfing ages toward outcrossing, thus increasing the mean number of heterozygous lethals in the younger selfing ages (black vs. green lines), corresponding to selective interference between recessive lethals and mildly deleterious mutations. But sufficiently many characters (red line) lower the purging threshold for quantitative genetic variance. At selfing rates above the purging threshold for quantitative genetic variance this creates a class of long-selfed lineages with improved fitness, purged of both lethals and quantitative genetic variance, which reduces both components of inbreeding depression and shifts the selfing age distribution back toward selfing. This qualitative shift in the equilibrium distribution of selfing ages also occurs for a given set of parameters when increasing the population selfing rate across the purging threshold for quantitative genetic variance in IMS and GAM without lethals (Lande and Porcher 2015), but not in K alone (Fig. 1), explaining why purging of quantitative genetic variance facilitates purging of lethals, but not vice versa. Qualitatively similar mechanisms operate in KGAM (not illustrated). 
in the absence of recessive lethals it produces two stable equilibria at selfing rates below the purging threshold for quantitative genetic variance (Lande and Porcher 2015). In combination with the Kondrashov model for lethals KGAM can thus create up to three alternative stable equilibria at a given selfing rate. Aside from the additional purged equilibrium that exists for quantitative genetic variance in KGAM at selfing rates below the purging threshold, and given that the purging threshold for quantitative genetic variance is much sharper in GAM than in IMS (Lande and Porcher 2015), the overall pattern of interaction of quantitative genetic variance with nearly recessive lethals resembles that in KIMS. That is, when the purging threshold for quantitative genetic variance alone exceeds that for recessive lethals alone, stabilizing selection on the quantitative characters interferes with purging of lethals; but when the purging threshold for quantitative genetic variance alone is less than that for recessive lethals alone, stabilizing selection on quantitative characters facilitates purging of lethals. Again, facilitation of purging recessive lethal mutations by stabilizing selection on quantitative traits becomes the dominant pattern of interaction with more characters and higher genomic mutation rates to lethals (Fig. 3 top row). Aside from the purged equilibrium that usually reflects purging in both the lethals and quantitative genetic variance, it appears that the main interaction is the influence of stabilizing selection on quantitative characters affecting the purging of lethals, whereas lethals have only a small impact on quantitative genetic variance. This interaction is again governed by the shape of the stable distribution of selfing ages among lineages, which with many characters is governed mainly by quantitative variation (Lande and Porcher 2015).

Further complications arise in KGAM at a high genomic mutation rate to lethals $(U=0.5,1)$ for an intermediate number of characters, such that two distinct purging thresholds exist for the equilibrium quantitative genetic variance which shifts back and forth between outcrossed and purged equilibria as the selfing rate increases. Results for these parameter values are plotted separately in the Supporting Information (Fig. S1).

\section{LIMIT CYCLES}

For a very narrow range of selfing rates in KIMS (only at $U=1, m=10, r \approx 0.54$ ), stable limit cycles (or strange attractors) appeared, involving a single cycle or double 
loop, with substantial sustained oscillations in mean lethals and quantitative genetic variance (Fig. 5). Changes in the total genetic variance in these cycles is entirely due to change in the negative covariance from linkage disequilibrium associated with oscillations in the age distribution of selfing lineages, since the genic variance at a given selfing rate remains constant in KIMS (see Appendix). The corresponding point in Fig. 2 represents the means of relevant variables averaged over the limit cycle.

In Kelly's (2007) approximate Kondrashov model structured by selfing age, limit cycles appeared as an artifact when the exact Kondrashov model produced transient damped oscillations at recombination rates near a purging threshold for lethals, where equilibrium deviations from a Poisson distribution of lethals are large (Lande et al. 1994). Damped oscillations also appeared in IMS and GAM near a purging threshold, due to linkage disequilibrium created by stabilizing selection magnified by inbreeding, and its breakdown by outcrossing and recombination (Lande and Porcher, unpublished results). The interaction of these oscillatory tendencies can create a limit cycle in KIMS, but this was not observed in KGAM.

[Fig. 5 about here]

\section{ROBUST RESULTS AND CONCLUSIONS}

Patterns of interaction between nearly recessive lethal mutations and stabilizing selection on quantitative characters that appear in both KIMS and KGAM models are considered to be robust results that do not depend on the particular details and approximations in either IMS or GAM (Lande and Porcher 2015). In the combined models strong interactions between nearly recessive lethals and additive genetic variance in quantitative characters occur for moderate to high genomic mutation rates to lethals and for weak stabilizing selection on multiple characters, such that in a randomly mating population at equilibrium the total inbreeding depression would be very high. We found three robust results.

(1) Alternative stable equilibria can exist for a given selfing rate over a considerable range of parameters. With a small number of characters under weak stabilizing selection, alternative equilibria are produced by the Kondrashov model for lethals alone. 
With many characters the interaction between lethals and quantitative characters creates or magnifies the difference between alternative equilibria.

(2) Stabilizing selection on quantitative characters usually exerts a stronger influence on nearly recessive lethals than vice versa. When the selfing rate at the purging threshold for quantitative genetic variance in IMS or GAM exceeds that for nearly recessive lethals in K, in the combined models, KIMS and KGAM, stabilizing selection on quantitative characters shows selective interference with the purging of lethals, augmenting the equilibrium mean number of lethals in comparison to K. Conversely when the selfing rate at the purging threshold for nearly recessive lethals in $\mathrm{K}$ exceeds that for quantitative genetic variance in IMS or GAM, in the combined models stabilizing selection causes selective facilitation in the purging of lethals, reducing the equilibrium mean number of lethals in comparison to K. Alternative equilibria at a given selfing rate, and the interactions between nearly recessive lethals and quantitative genetic variance, are mediated by shifts in the distribution of ages of selfing lineages in the population as illustrated for K alone (Fig. 1), for IMS and GAM (Lande and Porcher 2015), and in the combined models (Fig. 4).

(3) At high selfing rates above the purging threshold for quantitative genetic variance in KIMS and KGAM, only the purged equilibrium exists, with low quantitative genetic variance and low mean lethals. Purging of quantitative genetic variance at high selfing rates implies that highly selfing populations may fail to adapt to changing environments, thus supporting the hypothesis of Stebbins (1957) that predominant selfing is an "evolutionary dead end" (see discussion in Lande and Porcher 2015).

\section{LIMITATIONS OF THE MODELS}

Results that are specific to one model may not be robust and their realism remains uncertain. These include limit cycles in KIMS, the purged equilibrium for quantitative genetic variance and lethals at all selfing rates in KGAM, and multiple purging thresholds for quantitative genetic variance with intermediate numbers of characters in KGAM. Because IMS and GAM represent extremes of a range of possible models for the maintenance of quantitative genetic variance, the realism of these features must be explored using intermediate models with different assumptions and approximations. 
The main limitation of $\mathrm{K}$ is its assumption of no variance in the dominance coefficients among nearly recessive lethal mutations, and the difficulty of measuring this and the genomic mutation rate to lethals (Simmons and Crow 1977). Among mildly deleterious mutations, basic parameters of the joint distribution of heterozygous and homozygous effects on fitness, as well as their dominance, epistasis and linkage, and mutation rates, remain poorly understood due to statistical limitations afflicting empirical measurements (Eyre-Walker and Keightley 2007; Manolio et al. 2009; Yang et al. 2010). Investigating these complexities of the interaction between the two major components of inbreeding depression, represented in the bimodal distribution of fitness effects, appears to require numerical simulations that themselves may be limited to populations of small or moderate size dominated by random genetic drift (Bersabe et al. 2016), rather than by selection.

\section{ACKNOWLEDGMENTS}

We thank J. Kohn, B. Igic and the reviewers for helpful comments. This work was supported by a Royal Society Research Professorship and a grant from the Balzan foundation to R.L., by time on the computing cluster (UMS 2700 OMSI) at MNHN and CNRS program PICS grant \#5273 to E.P., and by Norwegian Research Council's Center of Excellence project SFF-III 223257. 


\section{LITERATURE CITED}

Arnold, S.J., R. Bürger, P.A. Hohenlohe, B.C. Ajie and J.G. Jones. 2008. Understanding the evolution and stability of the G-matrix. Evolution 62:2451-2461.

Bell, G. 2010. Experimental genomics of fitness in yeast. Proc. R. Soc. B. 277:14591467.

Bersabe, D., A. Caballero, A. Pérez-Figueroa and A. García-Dorado. 2016. On the consequences of purging and linkage on fitness and genetic diversity. G3 6:171181.

Bishir, J. and G. Namkoong. 1987. Unsound seeds in conifers: estimation of numbers of lethals and of magnitudes of effects associated with the maternal parent. Silvae Genetica 36:180-185.

Blows, M.W. 2006. A tale of two matrices: multivariate approaches in evolutionary biology. J Evol. Biol. 20:1-8.

Bobiwash, K., S.T. Schultz and D.J. Schoen. 2013. Somatic deleterious mutation rate in a woody plant: estimation from phenotypic data. Heredity 111:338-344.

Bulmer, M.G. 1971. The effect of selection on genetic variability. Am. Nat. 105:201211.

Burbidge, A.H. and S.H. James. 1991. Post-zygotic seed abortion in the genetic system of Stylidium (Angiospermae: Stylidiaceae). J. Heredity 82:319-328.

Campbell, R.B. 1986. The interdependence of mating structure and inbreeding depression. Theoret. Pop. Biol. 30:232-244.

Charlesworth, B. 2013a. Why we are not dead 100 times over. Evolution 67:3354-3361.

Charlesworth, B. 2013b. Stabilizing selection, purifying selection, and mutational bias in finite populations. Genetics 194:955-971.

Charlesworth, D. and B. Charlesworth. 1987. Inbreeding depression and its evolutionary consequences. Annu. Rev. Ecol. Syst. 18:237-268.

Charlesworth, D., and B. Charlesworth. 1995. Quantitative genetics in plants: the effect of the breeding system on genetic variability. Evolution 49:911-920.

Charlesworth, D., M. T. Morgan and B. Charlesworth. 1990. Inbreeding depression, genetic load, and the evolution of outcrossing rates in a multilocus system with no linkage. Evolution 44:1469-1489. 
Charlesworth, D. and J.H. Willis. 2009. The genetics of inbreeding depression. Nature Rev. Genet. 10:783-796.

Chevin, L.-M., M.E. Visser and J. Tufto. 2015. Estimating the variation, autocorrelation and environmental sensitivity of phenotypic selection. Evolution 69:23192332 .

Clayton, G. and A. Robertson. 1955. Mutation and quantitative variation. Am. Nat. $89: 151-158$.

Crow, J.F. and M. Kimura. 1970. An Introduction to Theoretical Population Genetics. Freeman, New York.

Dobzhansky, Th. 1970. Genetics of the Evolutionary Process. Columbia Univ. Press, New York.

Dorken, M.E. and B.C. Husband. 1999. Self-sterility in the understory herb Clintonia borealis (Liliaceae). Intl. J. Plant Sci. 160:577-584.

Engen, S., B.-E. Sæther, T. Kvalnes, and H. Jensen. 2012. Estimating fluctuating selection in age-structured populations. J. Evol. Biol. 25:1487-1499.

Eyre-Walker, A. and P.D. Keightley. 2007. The distribution of fitness effects of new mutations. Nat. Rev. Genet. 8:610-618.

Fisher, R. A. 1918. The correlation between relatives on the supposition of Mendelian inheritance. Trans. Roy. Soc. Edinb. 52:321-341.

Fisher, R. A. 1930. The Genetical Theory of Natural Selection. Clarendon Press, Oxford. [2nd edn. 1958. Dover, New York.]

Franklin, E.C. 1971. Estimates of frequency of natural selfing and inbreeding coefficients in loblolly pine. Silvae Genet. 20:194-195.

Fudala, A. and R. Korona. 2009. Low frequency of mutations with strongly deleterious but nonlethal fitness effects. Evolution 63:2164-2171.

Ganders, F.R. 1972. Heterozygosity for recessive lethals in homosporous fern populations: Theylpteris palustris and Onoclea sensibilis. Bot. J. Linn. Soc. 65:211-221.

Gibbs, P.E. 2014. Late-acting self-incompatibility-the pariah breeding system in flowering plants. New Phytol. 203:717-734.

Hadorn, E. 1961. Developmental Genetics and Lethal Factors. Wiley, New York.

Hao, Y.-Q., X.-F. Zhao, D.-Y. She, B. Xu, D.-Y. Zhang and W.J. Liao. 2012. The 
role of late-acting self-incompatibility and early-acting inbreeding depression in governing female fertility in monkshood, Aconitum kusnezoffii. PLoSONE 7:1-7.

Haldane, J.B.S. 1954. The measurement of natural selection. Proc. IX Intl. Cong. Genet. 1:480-487.

Husband, B. C. and D. W. Schemske. 1996. Evolution of the magnitude and timing of inbreeding depression in plants. Evolution 50:54-70.

Jarne, P. and J.R. Auld. 2006. Animals mix it up too: the distribution of selffertilization among hermaphroditic animals. Evolution 60:1816-1824.

Kelly, J.K. 2007. Mutation-selection balance in mixed mating populations. J. Theor. Biol. 246:355-365.

Kelly, J.K. 1999a. Response to selection in partially self-fertilizing populations. I. Selection on a single trait. Evolution 53:336-349.

Kelly, J.K. 1999b. Response to selection in partially self-fertilizing populations. II. Selection on multiple traits. Evolution 53:350-357.

Kimura, M. 1965. A stochastic model concerning the maintenance of genetic variability in quantitative characters. P. Natl. Acad. Sci. USA 54:731-736.

Kingsolver, J.G., H.E. Hoekstra, J.M. Hoekstra, D. Berrigan, S.N. Vignieri et al. 2001. The strength of phenotypic selection in natural populations. Am. Nat. 157:245261.

Kirkpatrick, M. 2009. Patterns of quantitative genetic variation in multiple dimensions. Genetica 136:271-284.

Klekowski, E.J., Jr. 1984. Mutational load in clonal plants: a study of two fern species Evolution 38:417-426.

Klekowski, E.J., Jr. 1988. Mutation, Developmental Selection, and Plant Evolution. Columbia Univ. Press, New York.

Klekowski, E.J., Jr. and and P.J. Godfrey. 1989. Ageing and mutation in plants. Nature 340:389-391.

Kondrashov, A.S. 1985. Deleterious mutations as an evolutionary factor. Facultative apomixis and selfing. Genetics 111:635-653.

Lande, R. 1975. The maintenance of genetic variability by mutation in a polygenic character with linked loci. Genet. Res. 26:221-235. 
Lande, R. 1977. The influence of the mating system on the maintenance of genetic variability in polygenic characters. Genetics 86:485-498.

Lande, R. 1980. Genetic variation and phenotypic evolution during allopatric speciation. Am. Nat. 116:463-479.

Lande, R. 2007. Expected relative fitness and the adaptive topography of fluctuating selection. Evolution 61:1835-1846.

Lande, R. 2008. Adaptive topography of fluctuating selection in a Mendelian population. J. Evol. Biol. 21:1096-1105.

Lande, R. and S. J. Arnold. 1983. The measurement of selection on correlated characters. Evolution 37:1210-1226.

Lande, R. and E. Porcher. 2015. Maintenance of quantitative genetic variance under partial self-fertilization, with implications for evolution of selfing. Genetics 200:891-906.

Lande, R. and D.W. Schemske. 1985. The evolution of self-fertilization and inbreeding depression in plants. I. Genetic models. Evolution 39:24-40.

Lande, R., D.W. Schemske and S.T. Schultz. 1994. High inbreeding depression, selective interference among loci, and the threshold selfing rate for purging recessive lethal mutations. Evolution 48:965-978.

Lande, R., and S. Shannon. 1996. The role of genetic variation in adaptation and population persistence in a changing environment. Evolution 50:434-437.

Manna, F., G. Martin and T. Lenormand. 2011. Fitness landscapes: an alternative theory for the dominance of mutation. Genetics 189:923-937.

Martin, G. and T. Lenormand. 2006. A general multivariate extension of Fisher's geometric model and the distribution of mutation fitness effects across species. Evolution 60:893-907.

Manolio, T.A, F.S. Collins, N.J. Cox, D.B. Goldstein, L.A. Hindorff et al. 2009. Finding the missing heritability of complex diseases. Nature 461:747-753.

McGlothlin, J.W. 2010. Combining selective episodes to estimate lifetime nonlinear selection. Evolution 64:1377-1385.

Muirhead, C.A. and R. Lande. 1997. Inbreeding depression under joint selfing, outcrossing and asexuality. Evolution 51:1409-1415. 
Porcher, E., and R. Lande. 2013. Evaluating a simple approximation to modeling the joint evolution of self-fertilization and inbreeding depression. Evolution 67:36283635 .

Porcher, E. and R. Lande. 2016. Inbreeding depression under mixed outcrossing, self-fertilization and sib-mating. BMC Evol. Biol. 16:105 (14pp).

Prout, T. 1965. The estimation of fitness from genotypic frequencies. Evolution 19:546551.

Prout, T. 1969. The estimation of fitness from population data. Genetics 63:949-967.

Roze, D. 2015. Effects of interference between selected loci on the mutation load, inbreeding depression, and heterosis. Genetics 201:745-757.

Schultz, S.T. and J.H. Willis. 1995. Individual variation in inbreeding depression: the roles of inbreeding history and mutation. Genetics 141:1209-1223.

Seavey, S.R. and K.S. Bawa. 1986. Late-acting self-incompatibility in Angiosperms. Bot. Rev. 52:195-219.

Scofield, D.G. and S.T. Schultz. 2006. Mitosis, stature and evolution of plant mating systems: low- $\Phi$ and high- $\Phi$ plants. Proc. R. Soc. B 273:275-282.

Siepielski, A.M., J.D. DiBattista, and S.M. Carlson. 2009. Its about time: the temporal dynamics of phenotypic selection in the wild. Ecol. Lett. 12:1261-1276.

Simmons, M. J., and J. F. Crow. 1977. Mutations affecting fitness in Drosophila populations. Annu. Rev. Genet. 11:49-78.

Simpson, G.G. 1953. The Major Features of Evolution. Columbia Univ. Press, New York.

Sorensen, F. 1969. Embryonic genetic load in coastal Douglas-fir, Pseudotsuga menziesii var. menziesii. Am. Nat. 103:389-398.

Sorensen, F. 1973. Frequency of seedlings from natural self-fertilization in coastal douglas-fir. Silvae Genet. 22:10-24.

Stebbins, G.L. 1957. Self fertilization and population variability in higher plants. Am. Nat. 91:337-354.

Vassilieva, L.L., A. M. Hook, and M. Lynch. 2000. The fitness effects of spontaneous mutations in Caenorhabditis elegans. Evolution 54:1234-1246.

Willis, J.H. 1992. Genetic analysis of inbreeding depression caused by chlorophyll- 
deficient lethals in Mimulus guttatus. Heredity 69:562-572.

Willis, J.H. 1999a. Inbreeding load, average dominance, and the mutation rate for mildly deleterious alleles in Mimulus guttatus. Genetics 153:1885-1898.

Willis, J.H. 1999b. The role of genes of large effect on inbreeding depression in Mimulus guttatus. Evolution 53:1678-1691.

Winn, A.A., E. Elle, S. Kalisz, P.-O. Cheptou, C.G. Eckert, et al. 2011. Analysis of inbreeding depression in mixed-mating plants provides evidence for selective interference and stable mixed mating. Evolution 65:3339-3359.

Wright, S. 1921. Systems of mating. II. The effects of inbreeding on the genetic composition of a population. Genetics 6:124-143.

Wright, S. 1931. Evolution in Mendelian populations. Genetics 16:97-159.

Wright, S. 1935. The analysis of variance and the correlations between relatives with respect to deviations from an optimum. J. Genet. 30:243-256.

Wright, S. 1968. Evolution and the Genetics of Populations. Vol. 1. Genetic and Biometric Foundations. Univ. Chicago Press, Chicago.

Wright, S. 1969. Evolution and the Genetics of Populations. Vol. 2. The Theory of Gene Frequencies. Univ. Chicago Press, Chicago.

Yang, J.A., B. Benyamin, B.P. McEvoy, S. Gordon, A.K. Henders et al. 2010. Common SNPs explain a large proportion of the heritability for human height. Nature Genet. 42:565-569.

\section{Supporting Information}

Additional Supporting Information may be found in the online version of this article at the publisher's website:

Recursion equations for IMS and GAM

Figure S1. Parameter values in KGAM producing two purging thresholds for the equilibrium quantitative genetic variance as a function of selfing rate. 
${ }_{818}$ Appendix

\section{MODIFICATION OF GENIC VARIANCE IN IMS}

The genic variance in infinitesimal model for selfing (IMS) is a limiting case of genetic variance maintained by purely additive mutational variance $\sigma_{m}^{2}$ in a finite population of effective size $N_{e}$ under random mating, $V(0)=2 N_{e}(0) \sigma_{m}^{2}$ (Clayton and Robertson 1955; Lande 1980), as $N_{e} \rightarrow \infty$ and $\sigma_{m}^{2} \rightarrow 0$ such that their product is a constant of the same order as the environmental variance in a quantitative character. Lande and Porcher (2015) extended this to a population with selfing rate $r$ and mean inbreeding coefficient $\bar{f}=r /(2-r)$ of Wright $(1921,1969)$ for a selective neutral partially selfing population.

Here, for increased accuracy when recessive lethals are included in the model, which can produce a high total inbreeding depression, we employ the actual mean inbreeding coefficient based on numerical calculation of the selfing age distribution,

$$
\bar{f}=\sum_{\tau=0}^{\infty} p_{\tau} f_{\tau}
$$

in concert with Wright's more general formula for the effective size of an inbred population with non-uniform inbreeding among individuals, $N_{e}(r)=N_{e}(0) /(1+\bar{f})$, such that at equilibrium for a given selfing rate

$$
V(r)=V(0) /(1+\bar{f}) .
$$

Numerical methods to achieve convergence to equilibrium are described in the Supporting Information. 
836 Table 1. Life cycle with notation for early selection on nearly recessive lethals and ${ }_{837}$ late selection on quantitative characters.

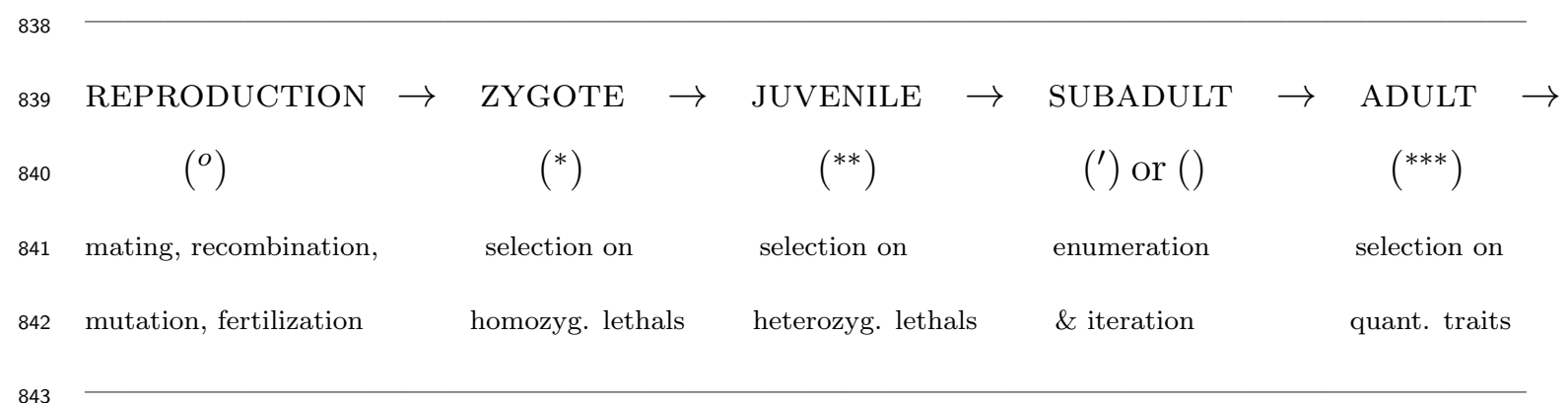


Figure 1. Alternative equilibria in model $\mathrm{K}$ alone, the Kondrashov model for lethals structured by selfing age, represented by solid and dashed lines. (A) Equilibrium mean number of heterozygous lethals $\bar{x}$, as a function of selfing rate $r$, with genomic mutation rate to lethals $U=0.5$ and dominance coefficient $h=0.02$. For alternative equilibria at selfing rate $r=0.4$, equilibrium probability distributions of number of heterozygous lethals (B) and selfing age (C).

Figure 2. Equilibrium mean number of heterozygous lethals $\bar{x}$, mean genetic variance $\bar{G}$, mean total fitness $\overline{\bar{v}} \overline{\bar{w}}$, total inbreeding depression $\delta$, and mean inbreeding coefficient $\bar{f}$ at equilibrium in KIMS as functions of selfing rate $r$ for different values of $U$. Colors of lines represent numbers of quantitative characters under stabilizing selection: $m=1$ (black), 10(blue),25(green),50(orange),100(red). Alternative equilibria are shown by solid and dashed lines. Parameters: $h=0.02$, equilibrium genetic variance under random mating $V(0)=1$, environmental variance $E=1$, width of individual fitness function $\omega^{2}=20$.

Figure 3. The same as Fig. 2 but for KGAM, where the mean genetic variance after selection on adults, $\bar{G}^{* * *}$ is plotted instead of $\bar{G}$ (Lande and Porcher 2015). Alternative equilibria are shown by solid, dashed and long-dashed lines. Parameters: $h=0.02$, mutational variance $\sigma_{m}^{2}=10^{-3}$, number of loci per character $n=10, E=1$, $\omega^{2}=20$.

Figure 4. Equilibrium total genetic variance $G$, mean number of heterozygous lethals $\bar{x}$, mean total fitness $\bar{v} \bar{w}$, and $\log _{10}$ frequency as functions of $\log _{10}$ selfing age in KIMS at selfing rate $r=0.2$ and $U=0.2$, for different number of characters $m=$ 1(black), 25(green), 100(red). Other parameters as in Fig. 2. Selfing age is plotted on a log scale to help reveal rapid changes in these quantities during the first few generations of continued selfing.

Figure 5. Limit cycles for mean number of heterozygous lethals and mean genetic 
variance $\bar{G}$ in KIMS. Points on the continuous curves represent sequential generations of a cycle of one or two loops. (A) $r=0.540$, (B) $r=0.543$. Blue and red colors in (B) distinguish the two loops. In successive cycles, the points would appear at slightly different positions on the curves. Other parameters: $U=1, h=0.02, m=10, V(0)=1$, $\omega^{2}=20$.

881 

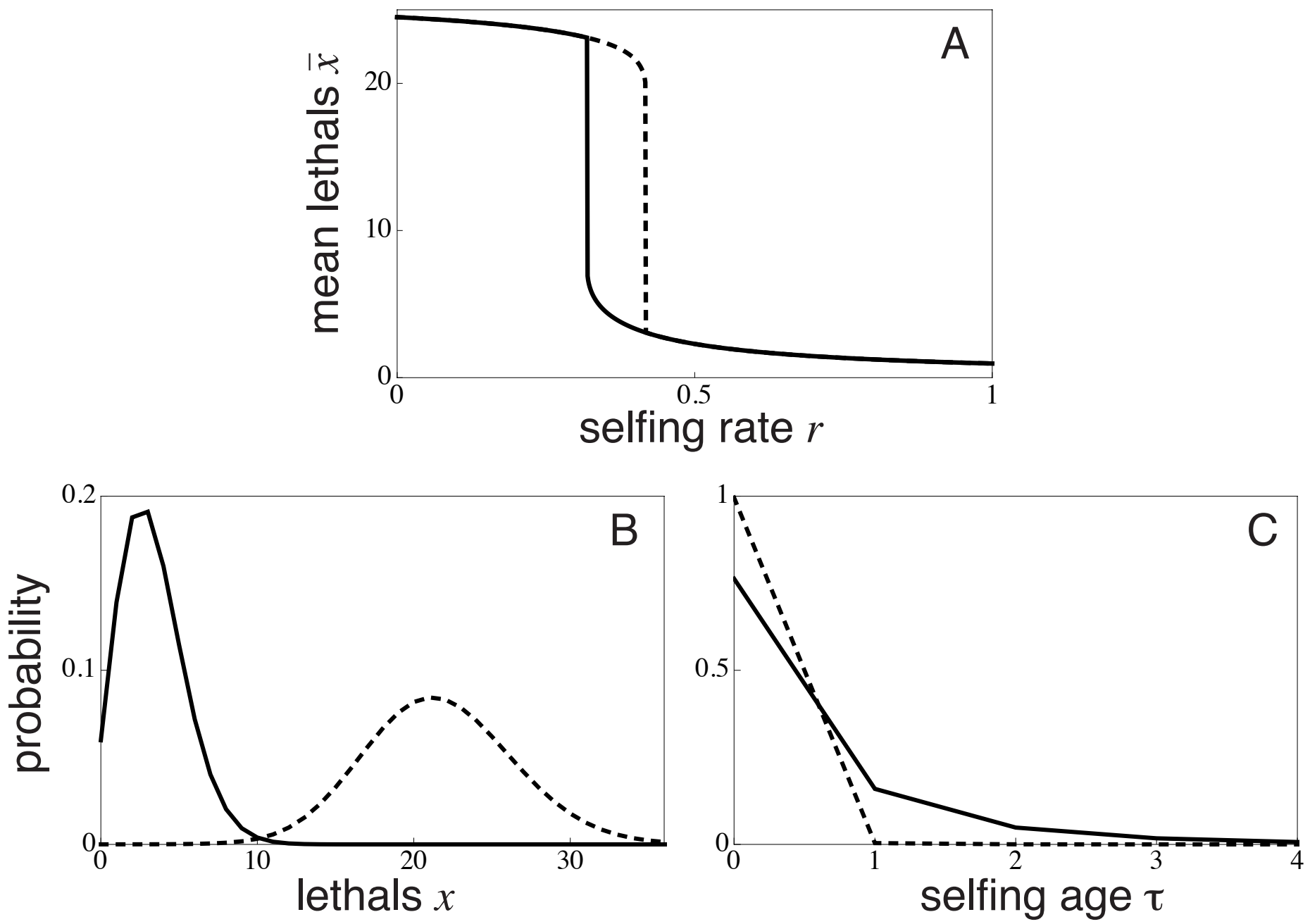

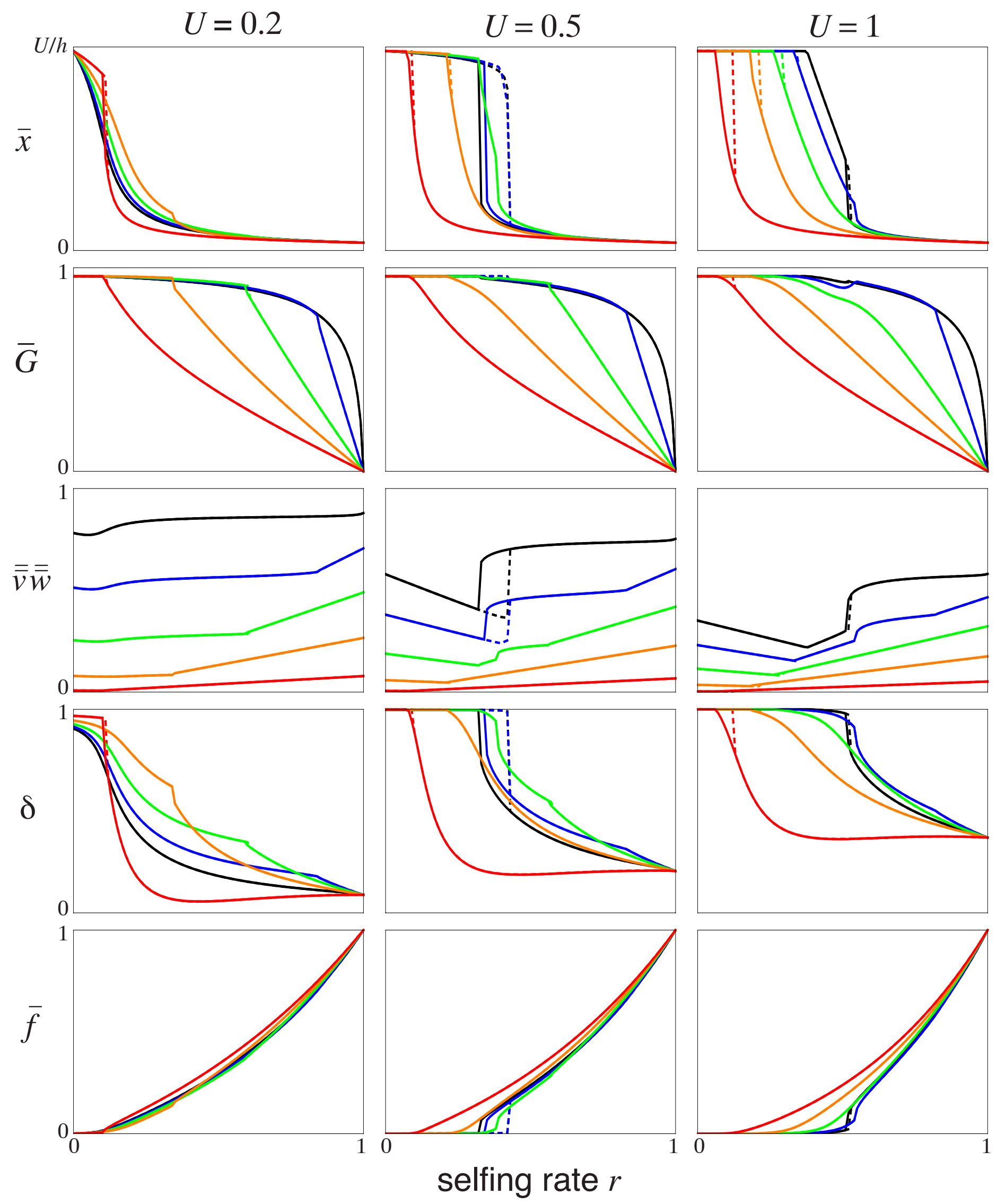

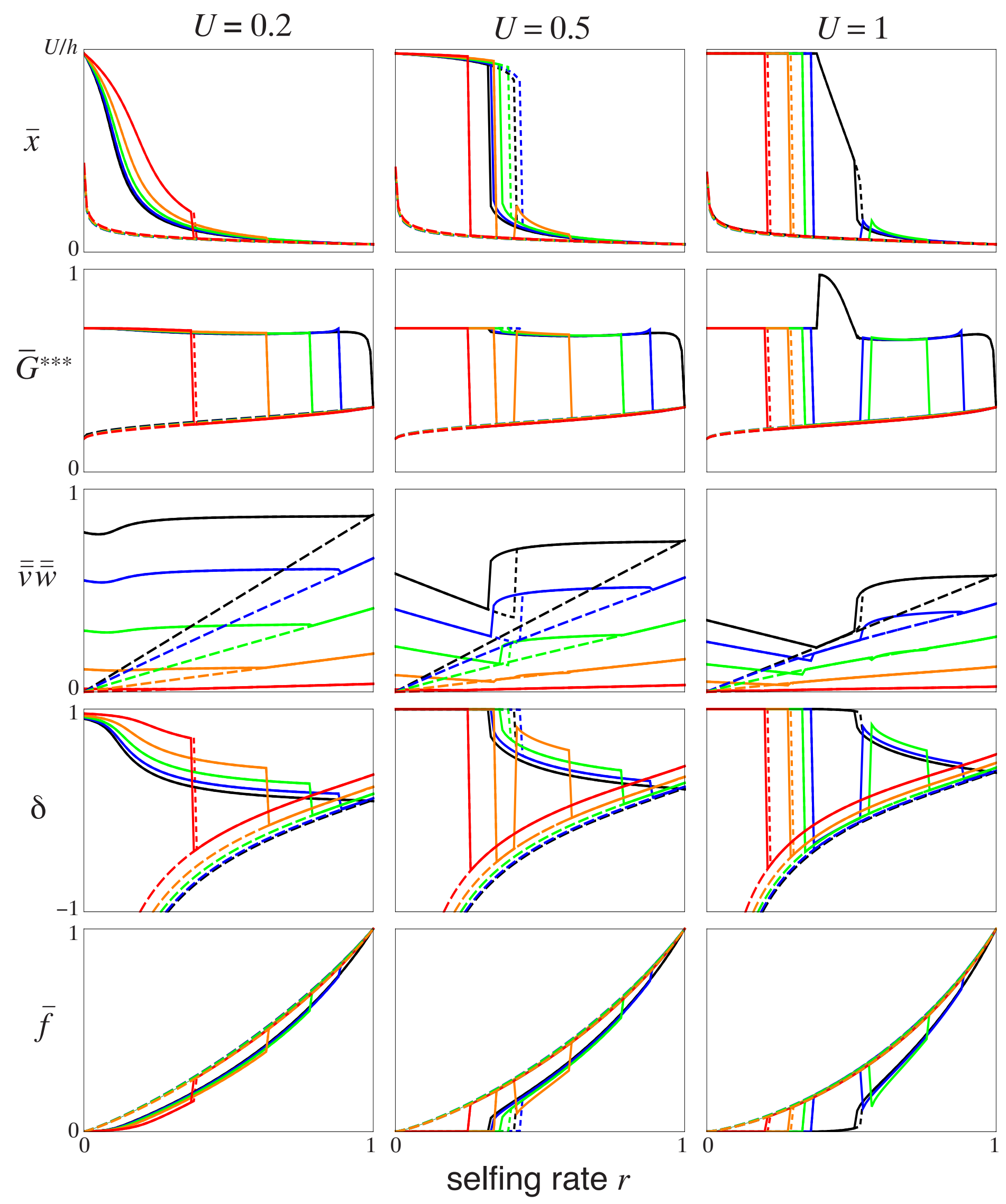

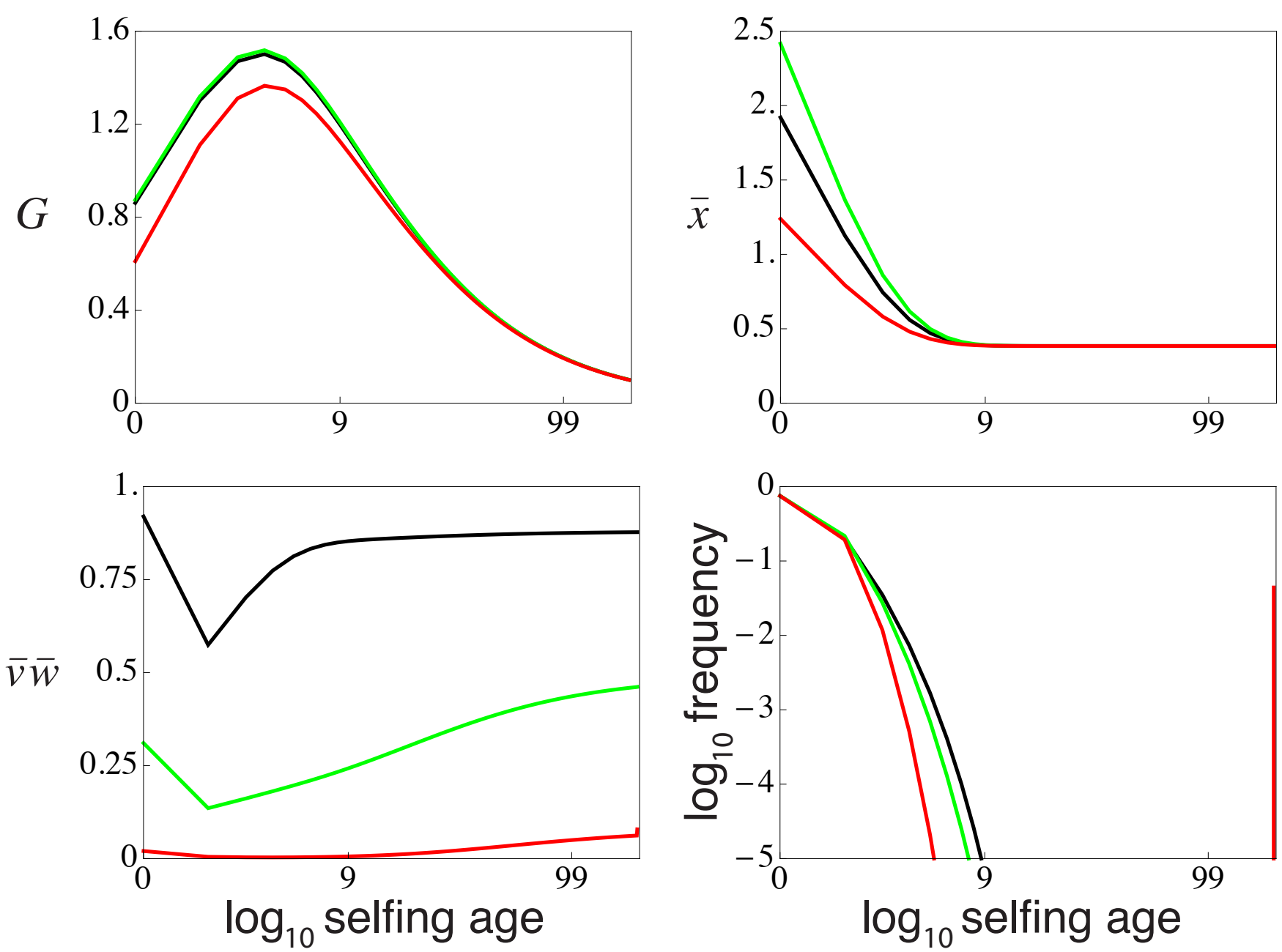


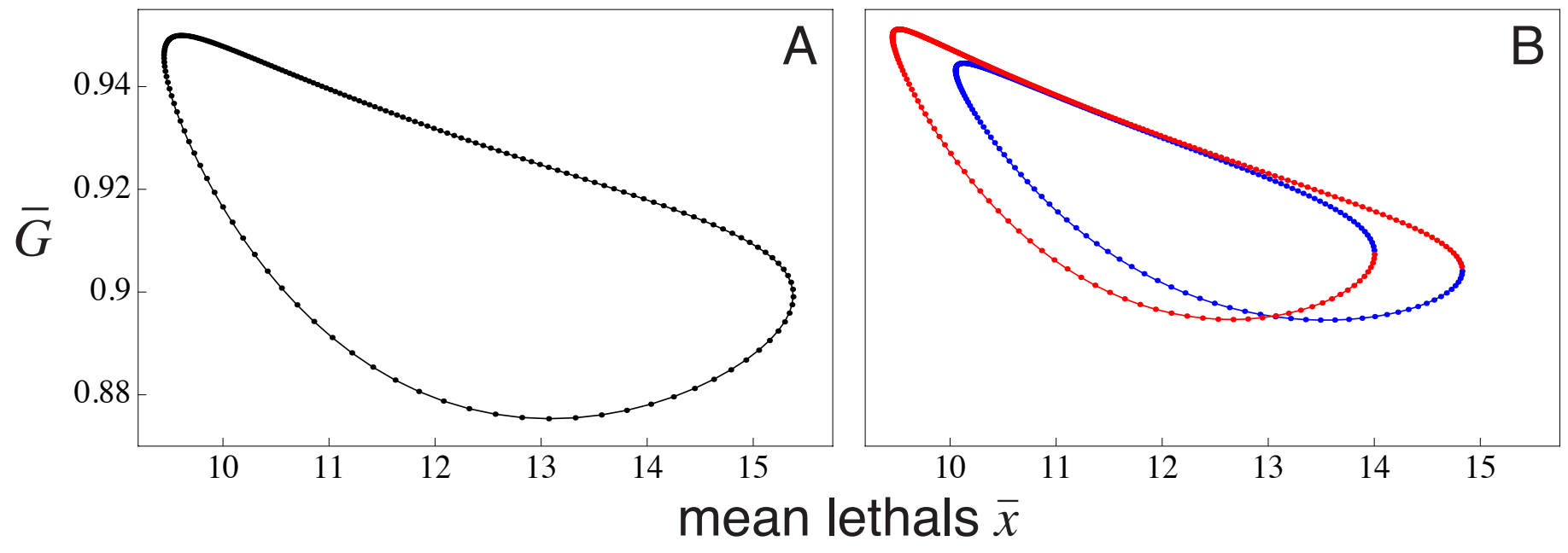

Lande \& Porcher Fig. 5 